\title{
Adipose-Derived Mesenchymal Stromal Cell-Derived Exosomes Alleviate Testicular Torsion Injury via Activation of PI3K/AKT and MAPK/ERK1/2 Pathways
}

\section{Hengchen Liu}

Second Affiliated Hospital of Harbin Medical University

\section{Manyu Shi}

Second Affiliated Hospital of Harbin Medical University

\section{Xiangqi Li}

Second Affiliated Hospital of Harbin Medical University

\section{Wenjun Lu}

Second Affiliated Hospital of Harbin Medical University

\section{Mingzhao Zhang}

Second Affiliated Hospital of Harbin Medical University

\section{Tingting Zhang}

Second Affiliated Hospital of Harbin Medical University

\section{Yang Wu}

Second Affiliated Hospital of Harbin Medical University

\section{Zenan Zhang}

Second Affiliated Hospital of Harbin Medical University

\section{Qingbo Cui}

Second Affiliated Hospital of Harbin Medical University

\section{Shulong Yang}

Second Affiliated Hospital of Harbin Medical University

\section{Zhaozhu Li ( $\nabla$ zhaozhu247@163.com )}

The Second Affiliated Hospital of Harbin Medical University https://orcid.org/0000-0002-8953-8186

\section{Research}

Keywords: Mesenchymal stromal cells, Exosomes, Testicular torsion, PI3K/AKT, MAPK/ERK1/2

Posted Date: September 17th, 2021

DOI: https://doi.org/10.21203/rs.3.rs-675580/v2 
License: (c) (i) This work is licensed under a Creative Commons Attribution 4.0 International License. Read Full License 


\section{Abstract}

Background: Exosomes have became a new therapeutic method in recent years. Adipose-derived mesenchymal stromal cell-derived exosomes (ADSC-Exos) have shown great potential in the treatment of ischemia-reperfusion injury. However, there is no research on whether ADSC-Exos can alleviate testicular torsion injury. Therefore, we investigated the protective effect of ADSC-Exos in alleviating testicular torsion-detorsion injury.

Methods: ADSC-Exos were isolated by ultracentrifugation and injected into the torsion-detorsion-affected testes of rats. H\&E staining and sperm quality were used to evaluate the therapeutic effects of ADSCExos, and the level of tissue oxidative stress was determined using malondialdehyde (MDA) and superoxide dismutase (SOD). In addition, TUNEL staining and immunohistological analyses (Ki67, cleaved Caspase-3, IL-6, IL-10, CCR7, and CD163) were used to clarify the effects of ADSC-Exos on spermatogenic cell proliferation, apoptosis, and the inflammatory microenvironment in vivo. Possible signaling pathways were predicted using sequencing technology and bioinformatics analysis. The signaling pathways were validated by proliferation (EdU), migration (transwell and scratch test), and apoptosis (flow cytometry, TUNEL, and western blot) in vitro.

Results: ADSC-Exos alleviated testicular torsion-detorsion injury by attenuating oxidative stress and inflammatory responses. In addition, ADSC-Exos promoted the proliferation and migration of spermatogenic cells and inhibited their apoptosis by activating the PI3K/AKT and MAPK/ERK1/2 signaling pathways.

Conclusions: Overall, we demonstrated that ADSC-Exos can alleviate testicular torsion injury and provided a new approach for treating testicular torsion injury.

\section{Introduction}

Testicular torsion is a urological emergency characterized by acute scrotal pain with nausea and vomiting, which often occurs in male children $[1,2]$. Early detection, early diagnosis, and early treatment are key to avoiding testicular necrosis $[3,4]$. The primary pathological mechanism of testicular torsion is ischemia-reperfusion (I/R) injury. Changes in microvascular blood flow cause the release of proinflammatory cytokines and production of reactive oxygen species (ROS) [5]. This leads to disorganization of the spermatogenic cell structure and function, or even apoptosis, leading to spermatogenic impairment [6, 7]. Thus, early surgery combined with antioxidants, anti-inflammatory cytokines, or other drugs is an important means of improving the prognosis of testicular torsion.

Adipose-derived mesenchymal stromal cells (ADSCs) play a profound role in preclinical studies [8, 9]. Abundant data have demonstrated that ADSCs have many roles in the treatment of organ I/R injury, such as anti-inflammatory, antioxidant, and anti-apoptotic roles [10-13]. Local injection of ADSCs has been shown to rescue testicular torsion-induced infertility $[14,15]$. However, recent studies have found that transplanted mesenchymal stromal cells (MSCs) do not survive effectively in the ischemic 
microenvironment post infarction [16]. Therefore, the functional benefits of MSC transplantation are likely due to the release of paracrine mechanisms, such as exosomes, which can regulate cell growth [17]. Recent studies indicate that exosomes derived from ADSCs (ADSC-Exos) can effectively reduce I/R injury in the brain [18], heart [19], and kidney [12]. However, there is no research on whether ADSC-Exos can alleviate testicular I/R injury. We hypothesized that ADSC-Exos could alleviate testicular torsion-detorsion injury. Specifically, we evaluated the effect of ADSC-Exos on spermatogenic cell viability, sperm quality, and inflammation in vivo, and the proliferation, migration, and apoptosis of spermatogenic cells in vitro.

\section{Materials And Methods}

\section{Animals}

Male Sprague-Dawley rats (200-250 g) at 8-10 weeks of age were purchased from the Animal Experiment Center of Harbin Medical University. All experiments involving animals were approved by the Ethics Committee of Harbin Medical University (no. Ky2018-135).

\section{Isolation and identification of ADSCs and ADSC-Exos}

The ADSCs and ADSC-Exos were isolated as in our previous study [20]. Briefly, ADSCs were isolated from subcutaneous fat and cultured in DMEM/F12 (Sigma, MO, USA), supplemented with 10\% FBS (Sigma), and $1 \%$ penicillin and streptomycin (Beyotime, Haimen, China) at $37{ }^{\circ} \mathrm{C}$ with $5 \% \mathrm{CO} 2$. The culture medium of ADSCs at passage three was replaced with exosome-depleted medium for $24 \mathrm{~h}$ and collected to isolate ADSC-Exos by ultracentrifugation, as previously reported [20]. The surface markers (CD90, CD105, CD34, CD45, CD11b), and multilineage differentiation capacity (adipogenic, osteogenic, and chondrogenic) of ADSCs have been demonstrated in our previous study [20]. Nanoparticle tracking analysis, transmission electron microscopy (TEM), and western blotting were used to identify the collected exosomes.

\section{Experimental protocols and surgical procedures}

Sixty rats were randomly divided into three groups: (1) Control $(n=12)$ : animals received surgery for scrotal incision and suturing, (2) l/R ( $n=24)$ : animals received surgery for testicular torsion and local injection of $100 \mu \mathrm{L}$ PBS before detorsion, (3) ADSC-Exos $(n=24)$ : animals received a local injection of $100 \mu \mathrm{L}$ PBS containing $400 \mu \mathrm{g}$ ADSC-Exos before detorsion.

All surgical procedures were performed under aseptic conditions by anesthesia with ketamine (50 $\mathrm{mg} / \mathrm{kg}$ ). The left testis was rotated $720^{\circ}$ in the anticlockwise direction for $3 \mathrm{~h}$. After that, the testis was detached via the same surgical approach. Half an hour before detorsion, $100 \mu \mathrm{L}$ ADSC-Exos or PBS was injected into the testis. The animals were sacrificed on the third and seventh postoperative days to collect the testes and epididymides for further study.

\section{Determination of spermatozoal parameters}


Rat epididymal tissues were cut into $1 \mathrm{~mm} 3$ cubes and immersed in $0.9 \% \mathrm{NaCl}$ at $37{ }^{\circ} \mathrm{C}$ for $20 \mathrm{~min}$ to extract the spermatozoa. Sperm quality (quantity, morphology, and motility) was assessed using the WHO sperm analysis method [21]. The morphology and motility of 200 sperm in each group were evaluated.

\section{Histopathological and immunohistological analyses}

Testicular tissues were fixed in Davidson's fixative (Beyotime) and the tissue sections were stained with hematoxylin and eosin (H\&E). Johnsen's score (Table 1) was used to evaluate the spermatogenic function [22]. Fifty seminiferous tubules were examined in each testis.

The tissue sections were immunohistochemically stained by primary antibody anti-Ki-67 (Affinity Biologicals, ON, Canada), followed by incubation with goat anti-rabbit IgG secondary antibody (Abcam, Cambridge, UK). For immunofluorescence analysis, the tissue sections were incubated with primary antibodies anti-cleaved Caspase-3 (Abcam), anti-CCR7 (Abcam), anti-CD163 (Abcam), anti-IL-6 (Origene), and anti-IL-10 (Abcam), followed by incubation with secondary antibodies (Abcam). Nuclei were stained with 4',6-diamidino-2-phenylindole (DAPI). Positive signals were quantified using Image $\mathrm{J}$ software.

\section{Biochemical analysis}

The malondialdehyde (MDA) in testicular tissue was determined by colorimetry measurement using the Lipid Peroxidation MDA Assay Kit (Beyotime). Superoxide dismutase (SOD) in testicular tissue was detected using the Cu/Zn-SOD, and Mn-SOD assay kits with WST-8 (Beyotime).

\section{Cell culture and treatment}

The GC-1 spg cell line was purchased from the American Type Culture Collection (ATCC, Manassas, VA, USA). The cells were cultured in DMEM with high glucose (Sigma) supplemented with $10 \%$ FBS, and $1 \%$ penicillin and streptomycin at $37^{\circ} \mathrm{C}$ with $5 \%$ CO2. To establish the l/R model in vitro, $1 \times 106$ cells were cultured in glucose-free DMEM (Sigma) in a $1 \% 02$ environment for $18 \mathrm{~h}$, followed by reoxygenation with normal 02 in complete medium with or without exosomes.

In vitro, we randomly sorted GC-1 spg cells into six groups as follows: (1) Control: cells cultured under normal conditions, (2) I/R: induction of cellular I/R injury only, (3) ADSC-Exos (100): induction of cellular $\mathrm{I} / \mathrm{R}$ injury, and reoxygenation with $100 \mu \mathrm{g} / \mathrm{mL}$ ADSC-Exos, (4) ADSC-Exos (200): reoxygenation with 200 $\mu \mathrm{g} / \mathrm{mL}$ ADSC-Exos, (5) ADSC-Exos+LY: pretreated with $50 \mu \mathrm{M}$ LY294002 (PI3K/AKT inhibitor, MedChemExpress, NJ, USA) for 30 min before reoxygenation with $200 \mu \mathrm{g} / \mathrm{mL}$ ADSC-Exos, (6) ADSCExos+PD: pretreated with $50 \mu \mathrm{M}$ PD 98059 (MAPK/ERK1/2 inhibitor, MedChemExpress) for 30 min before reoxygenation with $200 \mu \mathrm{g} / \mathrm{mL}$ ADSC-Exos. Cells from each group were collected after $30 \mathrm{~min}$ or $24 \mathrm{~h}$ for western blotting, after $3 \mathrm{~h}$ for flow cytometric analysis or the TUNEL assay, and after $24 \mathrm{~h}$ for EdU, transwell, and scratch assays.

\section{ADSC-Exos internalization analysis}


ADSC-Exos were labeled using PKH26 dye (Sigma). After ultracentrifugation, PKH-26 labeled ADSC-Exos were added to GC-1 spg cells cultured in exosome-depleted medium. The nuclei were counterstained with DAPI after $24 \mathrm{~h}$, and the internalization of ADSC-Exos was observed under a fluorescence microscope.

\section{Proliferation of GC-1 spg cells}

For cell proliferation analysis, $10 \mu \mathrm{M}$ 5-ethynyl-2-deoxyuridine (EdU) was added to the GC-1 spg cell culture medium for $30 \mathrm{~min}$. Then, the cells were fixed and stained using an EdU assay kit (UE, China). Next, cell proliferation was observed under a fluorescence microscope after DAPI counterstaining of the nuclei.

\section{Migration of GC-1 spg cells}

For cell migration analysis, we chose the scratch test and transwell assays. Briefly, after hypoxic injury with a serum-free culture medium, a scratch was made through the cultured cells. The extent of cell migration was measured after 0 and $24 \mathrm{~h}$.

Each group of cells after hypoxic injury was digested for transwell assays. A total of $1 \times 105 \mathrm{GC}-1 \mathrm{spg}$ cells were cultured in the upper chambers. The reoxygenation medium was added to the lower chambers. The upper chamber was fixed with paraformaldehyde and stained with crystal violet after $24 \mathrm{~h}$.

\section{Western blot analysis}

Cells were lysed in RIPA buffer (Beyotime) to extract the proteins. Immunoblotting was performed with the primary antibodies, anti-Hsp70, anti-TSG101, anti-CD9, anti-AKT, anti-phospho-AKT, anti-ERK1/2, antiphospho-ERK1/2, anti-Bcl-2, anti-Bax, and anti- $\beta$-actin (all from Abcam), according to the manufacturer's instructions. Goat anti-rabbit IgG (Abcam) was used as the secondary antibody. Image $\mathrm{J}$ software was used for quantification of protein bands.

\section{Flow cytometry analysis}

To analyze the apoptosis, cells were collected and stained with the FITC Annexin V Apoptosis Detection Kit (Becton-Dickinson, USA), and then analyzed by flow cytometry. Becton-Dickinson in-house FACSDiva software was used to analyze the data.

\section{Terminal deoxynucleotidyl transferase dUTP nick end labeling (TUNEL) assay}

In vivo, apoptosis of spermatogenic cells was detected using a TUNEL apoptosis assay kit (Wanleibio, Shenyang, China). Briefly, paraffin sections of testicular tissues were incubated with $50 \mu \mathrm{L}$ TUNEL reaction mixture. The sections were dehydrated and fixed after hematoxylin counterstaining of the nuclei. Apoptosis of spermatogenic cells was evaluated under a light microscope.

In addition, we performed a TUNEL assay in vitro. Briefly, GC-1 spg cells from each group were fixed and stained with a TUNEL Assay Apoptosis Detection Kit (UE, China). Next, apoptosis was observed under a 
fluorescence microscope after DAPI counterstaining of the nuclei.

\section{Micro RNA (miRNA) sequencing and data analysis}

The sequencing of miRNA in ADSC-Exos was performed by OE Biotech Company (Shanghai, China). Briefly, 20 ng of exosomal RNA was extracted and sequenced using an Illumina HiSeq 2500 instrument (Illumina, CA, USA) $(n=3)$. The top 50 highly expressed miRNAs in ADSC-Exos were predicted to target genes using miRanda software. Gene Ontology (GO) and Kyoto Encyclopedia of Genes and Genomes (KEGG) pathway enrichment analyses of target genes were performed using DAVID (https://david.ncifcrf.gov/), and KOBAS 3.0 (http://kobas.cbi.pku.edu.cn/kobas3/), respectively. The results of the analyses were visualized using $\mathrm{R}$ software.

\section{Statistical analysis}

Data were expressed as the mean \pm standard deviation (SD). Statistical analysis for multiple groups were conducted by the Tukey-Kramer $\mathrm{t}$-test. $\mathrm{P}<0.05$ was considered statistically significant.

\section{Results}

\section{Characterization of ADSC-Exos}

ADSC-Exos exhibited circular vesicular structures on TEM (Figure 1A). The size of ADSC-Exos was concentrated at $111.9 \mathrm{~nm}$ (Figure 1B). Western blot analyses demonstrated high expression of surface markers of ADSC-Exos, including CD9, TSG101, and HSP70 (Figure 1C).

\section{Alleviation of testicular torsion-detorsion injury by ADSC-Exos}

H\&E staining showed that normal testes showed normal testicular structure, normal morphology of seminiferous tubules, and many mature sperm. However, $3 \mathrm{~d}$ after torsion-detorsion injury, severe injuries were observed in the testes, which manifested as seminiferous tubule disorders, unclear boundaries, interstitial edema, and few sperm. In contrast, the histological appearance of the testes was significantly improved after treatment with ADSC-Exos. Seven days after torsion-detorsion injury, the ADSC-Exostreated group had significantly more spermatogenic cells compared with the IR group, and there was a more orderly arrangement (Figure 2A). The spermatogenic function was quantified using Johnsen's score and was substantially improved in the ADSC-Exos group on days 3 and 7 (Figure 2B).

To further determine whether ADSC-Exos could improve spermatogenesis after torsion-detorsion injury, we extracted sperm from the epididymis of each rat. Analysis of sperm parameters showed that testicular torsion-detorsion led to poor sperm quality. The quantity, mobility, and morphology of sperm were significantly decreased in the I/R group. However, treatment with ADSC-Exos significantly improved the sperm quality (Figure 2C-F). Furthermore, the level of MDA in the I/R group was significantly increased, while the level of SOD was decreased. ADSC-Exos treatment reduced MDA levels and increased SOD levels (Figure 2G, H). 


\section{Protection of spermatogenic cell activity by ADSC-Exos}

We next investigated whether ADSC-Exos affected spermatogenic cell activity after testicular torsiondetorsion injury. Immunohistochemistry revealed that the expression of Ki67 in spermatogenic cells was decreased after testicular torsion-detorsion injury. However, treatment with ADSC-Exos significantly increased the number of Ki67+ spermatogenic cells (Figure 3A). Apoptosis marker TUNEL staining revealed massive spermatogenic cell apoptosis after testicular torsion-detorsion injury. The number of apoptotic spermatogenic cells in the ADSC-Exos group on days 3 and 7 was lower than that in the I/R group (Figure 3B). As expected, cleaved Caspase-3 (a marker of apoptosis) immunofluorescence staining showed the same results as TUNEL staining (Figure 3C). ADSC-Exos therefore promote spermatogenic cell proliferation and reduce apoptosis after testicular torsion-detorsion injury (Figure 3D).

\section{The miRNA sequencing and bioinformatics analysis of ADSC-Exos}

To further understand how ADSC-Exos alleviate testicular torsion-detorsion injury, we sequenced the miRNA in ADSC-Exos. The top 50 miRNAs detected in ADSC-Exos are shown in Figure 4A. Next, we performed functional enrichment analysis of their possible target genes using GO and KEGG pathway enrichment analyses (Figure 4B). The biological process (BP) was mainly enriched in "regulation of cell adhesion", the cellular component (CC) was mainly enriched in "proteinaceous extracellular matrix", the molecular function (MF) was mainly enriched in "SH3 domain binding" (Figure 5A). KEGG pathway enrichment analyses showed that "PI3K/AKT", and "MAPK" were the main signaling pathways (Figure 5B). Therefore, we made a bold assertion that ADSC-Exos alleviate testicular torsion-detorsion injury via the PI3K/AKT, and MAPK/ERK1/2 signaling pathways and proceeded to test this hypothesis.

\section{ADSC-Exos activated the PI3K/AKT and MAPK/ERK1/2 signaling pathways}

To confirm our hypothesis, we added ADSC-Exos to GC-1 spg cells after I/R injury. We found that PKH-26 labeled ADSC-Exos could be internalized by GC-1 spg cells (Figure 6A). Western blot analyses proved that the p-AKT and p-ERK1/2 were decreased in GC-1 spg cells subjected to IR injury, and ADSC-Exos activated the PI3K/AKT and MAPK/ERK1/2 pathways. Furthermore, pretreatment with LY294002 and PD98059 inhibited the expression of p-AKT and p-ERK1/2, respectively (Figure 6B-E).

\section{ADSC-Exos regulated GC-1 spg cell proliferation and migration by activating PI3K/AKT and MAPK/ERK1/2 signaling pathways}

We evaluated the effects of ADSC-Exos on the proliferation and migration of GC-1 spg cells using different concentrations of ADSC-Exos, as well as pathway inhibitors. The EdU assays showed that ADSC-Exos promoted GC-1 spg cell proliferation after I/R injury in a dose-dependent manner, and LY294002 and PD98059 significantly attenuated this effect (Figure 7A, D). Similarly, the transwell assays (Figure 7B, E), and scratch test (Figure 7C, F) showed that ADSC-Exos promoted GC-1 spg cell migration after I/R injury, which could also be suppressed by LY294002 and PD98059. 


\section{ADSC-Exos protected GC-1 spg cells against apoptosis by activating PI3K/AKT and MAPK/ERK1/2 pathways signaling pathways}

Flow cytometry and TUNEL assays were used to detect apoptosis in the GC-1 spg cells. After I/R injury, the number of apoptotic cells in GC-1 spg cells significantly increased, whereas treatment with ADSCExos clearly decreased the apoptosis of cells. LY294002 and PD98059 inhibited the anti-apoptotic effects of ADSC-Exos (Figure 8A, B, F, J). In addition, western blotting showed that ADSC-Exos could increase the low expression of Bcl-2, and decrease the high expression of Bax caused by I/R. Similarly, LY294002 and PD98059 attenuated the regulation of Bcl-2 and Bax expression by ADSC-Exos (Figure 8C-E, G-I).

\section{ADSC-Exos regulate the inflammatory response induced by testicular torsion-detorsion injury}

We next investigated whether ADSC-Exos affected the early inflammatory response after testicular torsion injury. Immunofluorescence showed that a large amount of IL-6 (a pro-inflammatory factor) aggregated within the testicular tissue on day 3 after testicular torsion-detorsion injury and decreased on day 7 (Figure 9A). However, compared with the I/R group, both ADSC-Exos groups were significantly decreased on days 3 and 7. In contrast, IL-10 (an anti-inflammatory factor) was increased in the ADSC-Exos group (Figure 9B). In addition, we found that CCR7+ (M1 macrophage marker), and CD163+ (M2 macrophage marker) cells increased after testicular torsion-detorsion injury. However, compared with the I/R group, there were significantly less CCR7+ cells in the ADSC-Exos group, and conversely, CD163+ cells were significantly increased (Figure 9C, D). The results of the quantitative analyses are shown in Figure 9E.

\section{Discussion}

Testicular torsion is a major cause of testicular loss in male adolescents [3]. Effective anti-oxidation and anti-inflammatory adjuvant therapy are the main means of reducing I/R injury after testicular torsion. Recent studies have shown that ADSC-Exos can effectively alleviate I/R injury of the cerebrum and myocardium [23]. In this study, we found that ADSC-Exos can reduce oxidative stress, inhibit inflammation, promote proliferation and migration, and prevent apoptosis in the testes.

The physiological properties of spermatogenic cells, which are on the borderline of hypoxia, make them sensitive to changes in blood flow [24]. In the present study, the rat testes were severely damaged after I/R injury, whereas local injection of ADSC-Exos significantly improved the pathological features of testes. The ROS induced by I/R exceed the scavenging ability of antioxidant enzymes, leading to spermatogenic cell apoptosis. MDA is the end product of ROS and is a reliable indicator of the level of ROS [25]. SOD protects cells against superoxide radical damage by dismutating superoxide radicals into $\mathrm{H} 2 \mathrm{O} 2$ and 02 [26]. Although antioxidants can reduce the level of oxidative stress induced by testicular I/R injury [2729], antioxidants alone cannot improve spermatogenesis after injury [15]. Fortunately, transplantation of the uncultured adipose-derived stromal vascular fraction can promote spermatogenesis while reducing oxidative stress levels after testicular torsion [30]. As expected, in the present study, ADSC-Exos significantly reduced MDA levels, increased SOD levels, and improved sperm quality (quantity, morphology, and motility) after testicular torsion injury. 
After testicular torsion-detorsion injury, ROS production is accompanied by the activation of apoptotic pathways [31]. Apoptosis induced by testicular torsion-detorsion injury occurs in all spermatogenic cells, of which apoptosis of primary spermatocytes is the main reason for impaired fertility [32]. In the present study, TUNEL staining and cleaved Caspase-3 immunofluorescence analyses indicated that after testicular torsion injury, the caspase-dependent apoptosis pathways were activated and spermatogenic cells were largely apoptotic, whereas treatment with ADSC-Exos significantly attenuated the degree of apoptosis in spermatogenic cells. Seven days after injury, the number of apoptotic cells significantly decreased, indicating that apoptotic spermatogenic cells had died. However, H\&E staining indicated that the number of spermatogenic cells increased after ADSC-Exos treatment. Therefore, we investigated the effects on the proliferation of spermatogenic cells. Ki67 is a cell proliferation marker. Immunohistochemistry showed that in normal testicular tissue, spermatogenic cell proliferation mainly occurred in primary spermatocytes. The proliferative capacity of primary spermatocytes was inhibited after testicular torsion-detorsion injury. Following treatment with ADSC-Exos, the expression of Ki67 in spermatogenic cells was obviously increased. Interestingly, we also found that the proliferative effect of ADSC-Exos on spermatogenic cells was not restricted to primary spermatocytes. These results suggest that ADSC-Exos inhibit spermatogenic cell apoptosis and promote their proliferation after testicular torsion-detorsion injury.

Since miRNAs are the main components through which exosomes function [33], we sequenced miRNAs in ADSC-Exos to understand how ADSC-Exos alleviate testicular torsion-detorsion injury. The target genes were predicted and subjected to bioinformatics analysis. The enrichment analyses showed that PI3K/AKT and MAPK pathways may play major roles. Therefore, we hypothesized that ADSC-Exos alleviate testicular torsion-detorsion injury by activating the PI3K/AKT and MAPK/ERK1/2 pathways. To confirm our hypothesis, we examined the association between ADSC-Exos and PI3K/AKT and MAPK/ERK1/2 signaling pathways. We found that ADSC-Exos upregulated the decreased expression of p-AKT and $\mathrm{p}$-ERK1/2 induced by I/R injury. In addition, ADSC-Exos promoted proliferation and migration and inhibited the apoptosis of GC- 1 spg cells after I/R injury. Pathway inhibitors (LY294002 and PD98059) were used to further investigate the roles of the PI3K/AKT and MAPK/ERK1/2 pathways in ADSC-Exos alleviated I/R injury. LY294002 and PD98059 effectively inhibited the expression of p-AKT and $\mathrm{p}$-ERK1/2, respectively, and blocked the protective effects of ADSC-Exos against GC-1 spg cell I/R injury. This supports the assertion that ADSC-Exos alleviated testicular torsion-detorsion injury by promoting activation of the PI3K/AKT and MAPK/ERK1/2 signaling pathways.

The inflammatory response is an important pathological mechanism of I/R injury [34]. ROS-induced redox changes lead to the release of inflammatory cytokines [35]. Sevil et al. reported that the expression of TNF-a and IL- 6 was increased, and that of IL-10 was decreased after testicular torsion [32]. Turner et al. found that TNF-a, IL-6, and IL-1 $\beta$ released by macrophages exacerbated inflammation in testicular torsion-detorsion injury [36]. Recent studies have confirmed that the polarization of macrophages determines their role in inflammation [37-39]. In addition, previous studies have confirmed that ADSCExos modulate macrophage polarization from the pro-inflammatory M1 to the anti-inflammatory M2 phenotype in vitro [40-42]. In the present study, treatment with ADSC-Exos reduced the number of CCR7 
+ M1 macrophages and increased the CD163 + M2 macrophages after testicular torsion injury. As we expected, the expression of the pro-inflammatory cytokine, IL-6, was decreased, whereas the antiinflammatory cytokine, IL-10, was increased after ADSC-Exos treatment. ADSC-Exos therefore alleviate inflammation induced after testicular torsion injury by modulating macrophages.

In general, exosomes are used to repair damaged tissues via intravenous injection. Because of the physiological blood-testis barrier, transplanted exosomes may not be able to enter the testis via the circulation. Thus, we chose local injections to transplant ADSC-Exos. In addition, exosomes have little immunogenicity compared to stromal cell transplantation. Especially in ethically relevant organs such as the testis, the transplantation of exosomes has more advantages than stromal cells.

There are some limitations to the current study. First, we only evaluated the early efficacy of ADSC-Exos in ameliorating testicular torsion-detorsion injury, and the long-term efficacy, such as fertility function, still requires further study. Second, the optimal dose for the treatment of testicular torsion-detorsion injury requires further study. Third, although we predicted and validated the pathway by which ADSC-Exos play a role by sequencing, the specific miRNA still needs to be identified in subsequent studies.

\section{Conclusions}

This study showed that ADSC-Exos can alleviate testicular torsion-detorsion injury by reducing oxidative stress and promoting M2 polarization of macrophages to inhibit inflammation. ADSC-Exos activated the $\mathrm{PI} 3 \mathrm{~K} / \mathrm{AKT}$ and MAPK/ERK1/2 pathways to promote proliferation and migration and inhibit apoptosis of spermatogenic cells. Our study raises the feasibility of ADSC-Exos in alleviating testicular torsiondetorsion injury and provides a new avenue for treating testicular torsion injury.

\section{Abbreviations}

ADSCs: Adipose-derived mesenchymal stem cells, ADSC-Exos: Adipose-derived mesenchymal stem cell derived exosomes, MDA: malondialdehyde, SOD: superoxide dismutase, ROS: reactive oxygen species, DMEM: Dulbecco's modified Eagle's medium, FBS: fetal bovine serum, TEM: Transmission electron microscopy, EdU: 5-ethynyl-2'-deoxyuridine, H\&E: Hematoxylin-eosin, TUNEL: Terminal deoxynucleotidyl transferase dUTP nick end labeling, DAPI: 4',6-diamidino-2-phenylindole, GO: Gene Ontology, KEGG: Kyoto Encyclopedia of Genes and Genomes

\section{Declarations}

\section{Acknowledgements}

We would like to acknowledge the reviewers for their helpful comments on this paper.

\section{Authors' contributions}


$\mathrm{HCL}, \mathrm{MYS}$ contributed to cytology experiment, animal experiments, $\mathrm{HCL}, \mathrm{XQL}$ contributed to data acquisition, data analysis, HCL, WJL and MZZ contributed to manuscript writing, TTZ, YW ,ZNZ and QBC provided experimental technical support and final approval of manuscript, SLY and ZZL took part in the experimental design, text revision, and final approval of manuscript.

\section{Author's information}

Not applicable

\section{Funding}

This study was supported by the National Natural Science Foundation of China $(81871837,81572117)$ and the Specialized Research Fund for Doctoral Programs in Colleges and Universities of China (20132307110007).

\section{Availability of data and materials}

The datasets used and/or analyzed during the current study are available from the corresponding author on reasonable request.

\section{Ethics approval and consent to participate}

The experimental protocol about animals was approved by the Harbin Medical University Ethics Committee.

\section{Consent for publication}

Not applicable

\section{Competing interests}

The authors declare that they have no conflicting interests.

\section{References}

1. Li XL, Lu SY, Liu TT, Zhang QM, Zhang WP, Jia LQ. The theoretical method and clinical application of testicular torsion. Int Urol Nephrol. 2020,52(6):1009-14.

2. Jacobsen FM, Rudlang TM, Fode M, Østergren PB, Sønksen J, Ohl DA, Jensen CFS. The Impact of Testicular Torsion on Testicular Function. World J Mens Health. 2020,38(3):298-307.

3. van Heurn LW, Pakarinen MP, Wester T. Contemporary management of abdominal surgical emergencies in infants and children. Br J Surg. 2014,101(1):e24-33.

4. Pogorelić Z, Mrklić I, Jurić I. Do not forget to include testicular torsion in differential diagnosis of lower acute abdominal pain in young males. J Pediatr Urol. 2013,9(6 Pt B):1161-5. 
5. Bozkurt M, Degirmentepe RB, Polat EC, Yildirim F, Sonmez K, Cekmen M, Eraldemir C, Otunctemur A. Protective effect of hydrogen sulfide on experimental testicular ischemia reperfusion in rats. $\mathrm{J}$ Pediatr Urol. 2020,16(1):40.e1-8.

6. Carden DL, Granger DN. Pathophysiology of ischaemia-reperfusion injury. J Pathol. 2000,190(3):25566.

7. Shokoohi M, Shoorei H, Soltani M, Abtahi-Eivari SH, Salimnejad R, Moghimian M. Protective effects of the hydroalcoholic extract of Fumaria parviflora on testicular injury induced by torsion/detorsion in adult rats. Andrologia. 2018,50(7):e13047.

8. Qiu G, Zheng G, Ge M, Wang J, Huang R, Shu Q, Xu J. Mesenchymal stem cell-derived extracellular vesicles affect disease outcomes via transfer of microRNAs. Stem Cell Res Ther. 2018,9(1):320.

9. Cui X, He Z, Liang Z, Chen Z, Wang H, Zhang J. Exosomes From Adipose-derived Mesenchymal Stem Cells Protect the Myocardium Against Ischemia/Reperfusion Injury Through Wnt/ $\beta$-Catenin Signaling Pathway. J Cardiovasc Pharmacol. 2017,70(4):225-31.

10. Yip HK, Chang YC, Wallace CG, Chang LT, Tsai TH, Chen YL, Chang HW, Leu S, Zhen YY, Tsai CY, Yeh $\mathrm{KH}$, Sun $\mathrm{CK}$, Yen $\mathrm{CH}$. Melatonin treatment improves adipose-derived mesenchymal stem cell therapy for acute lung ischemia-reperfusion injury. J Pineal Res. 2013,54(2):207-21.

11. Yip HK, Lee MS, Sun CK, Chen KH, Chai HT, Sung PH, Lin KC, Ko SF, Yuen CM, Liu CF, Shao PL, Lee FY. Therapeutic effects of adipose-derived mesenchymal stem cells against brain death-induced remote organ damage and post-heart transplant acute rejection. Oncotarget. 2017 Sep 30,8(65):108692-711.

12. Lin KC, Yip HK, Shao PL, Wu SC, Chen KH, Chen YT, Yang CC, Sun CK, Kao GS, Chen SY, Chai HT, Chang $\mathrm{CL}$, Chen $\mathrm{CH}$, Lee MS. Combination of adipose-derived mesenchymal stem cells (ADMSC) and ADMSC-derived exosomes for protecting kidney from acute ischemia-reperfusion injury. Int $\mathrm{J}$ Cardiol. 2016,216:173-85.

13. Pu CM, Liu CW, Liang CJ, Yen YH, Chen SH, Jiang-Shieh YF, Chien CL, Chen YC, Chen YL. AdiposeDerived Stem Cells Protect Skin Flaps against Ischemia/Reperfusion Injury via IL-6 Expression. J Invest Dermatol. 2017,137(6):1353-62.

14. Hsiao $\mathrm{CH}$, Ji AT, Chang CC, Cheng CJ, Lee LM, Ho JH. Local injection of mesenchymal stem cells protects testicular torsion-induced germ cell injury. Stem Cell Res Ther. 2015,6(1):113.

15. Hsiao CH, Ji AT, Chang CC, Chien MH, Lee LM, Ho JH. Mesenchymal stem cells restore the sperm motility from testicular torsion-detorsion injury by regulation of glucose metabolism in sperm. Stem Cell Res Ther. 2019,10(1):270.

16. Tang XL, Li Q, Rokosh G, Sanganalmath SK, Chen N, Ou Q, Stowers H, Hunt G, Bolli R. Long-Term Outcome of Administration of c-kit(POS) Cardiac Progenitor Cells After Acute Myocardial Infarction: Transplanted Cells Do not Become Cardiomyocytes, but Structural and Functional Improvement and Proliferation of Endogenous Cells Persist for at Least One Year. Circ Res. 2016,118(7):1091-105.

17. Song Y, Li Z, He T, Qu M, Jiang L, Li W, Shi X, Pan J, Zhang L, Wang Y, Zhang Z, Tang Y, Yang GY. M2 microglia-derived exosomes protect the mouse brain from ischemia-reperfusion injury via exosomal 
miR-124. Theranostics. 2019,9(10):2910-23.

18. Huang X, Ding J, Li Y, Liu W, Ji J, Wang H, Wang X. Exosomes derived from PEDF modified adiposederived mesenchymal stem cells ameliorate cerebral ischemia-reperfusion injury by regulation of autophagy and apoptosis. Exp Cell Res. 2018,371(1):269-77.

19. Chai HT, Sheu JJ, Chiang JY, Shao PL, Wu SC, Chen YL, Li YC, Sung PH, Lee FY, Yip HK. Early administration of cold water and adipose derived mesenchymal stem cell derived exosome effectively protects the heart from ischemia-reperfusion injury. Am J Transl Res. 2019,11(9):5375-89.

20. Liu H, Zhang M, Shi M, Zhang T, Lu W, Yang S, Cui Q, Li Z. Adipose-derived mesenchymal stromal cell-derived exosomes promote tendon healing by activating both SMAD1/5/9 and SMAD2/3. Stem Cell Res Ther. 2021,12(1):338.

21. World Health Organization. Laboratory manual for the examination and processing of human semen (5th ed.). World Health Organization. 2010,11-102

22. Johnsen SG. Testicular biopsy score count--a method for registration of spermatogenesis in human testes: normal values and results in 335 hypogonadal males. Hormones. 1970,1(1):2-25.

23. Zheng X, Hermann DM, Bähr M, Doeppner TR. The role of small extracellular vesicles in cerebral and myocardial ischemia-Molecular signals, treatment targets, and future clinical translation. Stem Cells. 2021,39(4):403-13.

24. Creasy DM. Pathogenesis of male reproductive toxicity. Toxicol Pathol. 2001,29(1):64-76.

25. Zhang X, Lv F, Tang J. Protection from ischemia by preconditioning, postconditioning, and combined treatment in rabbit testicular ischemia reperfusion injury. Arch Biochem Biophys. 2016,608:1-7.

26. Filho DW, Torres MA, Bordin AL, Crezcynski-Pasa TB, Boveris A. Spermatic cord torsion, reactive oxygen and nitrogen species and ischemia-reperfusion injury. Mol Aspects Med. 2004,25(1-2):199210.

27. Wei SM, Huang YM, Zhou J. Probucol Reduces Testicular Torsion/Detorsion-Induced Ischemia/Reperfusion Injury in Rats. Oxid Med Cell Longev. 2017,2017:5424097.

28. Turkmen S, Mentese A, Karaguzel E, Karaca Y, Kucuk A, Uzun A, Yulug E, Turedi S. A comparison of the effects of $\mathrm{N}$-acetylcysteine and ethyl pyruvate on experimental testicular ischemia-reperfusion injury. Fertil Steril. 2012,98(3):626-31.

29. Erol B, Sari U, Amasyali AS, Ozkanli S, Sogut S, Hanci V, Efiloglu O, Danacioglu YO, Engin P, Yencilek F, Atis G, Yildirim A, Alkoc OA, Caskurlu T. Comparison of combined antioxidants and thymoquinone in the prevention of testis ischemia - reperfusion injury. Andrology. 2017,5(1):119-24.

30. Zhou L, Song K, Xu L, Zhao F, Tian H, Zhou C, Xu Z, Ge Y, Wu R, Jia R. Protective Effects of Uncultured Adipose-Derived Stromal Vascular Fraction on Testicular Injury Induced by Torsion-Detorsion in Rats. Stem Cells Transl Med. 2019,8(4):383-91.

31. Lysiak JJ, Nguyen QA, Kirby JL, Turner TT. Ischemia-reperfusion of the murine testis stimulates the expression of proinflammatory cytokines and activation of c-jun N-terminal kinase in a pathway to Eselectin expression. Biol Reprod. 2003,69(1):202-10. 
32. Arabaci Tamer S, Yildirim A, Köroğlu MK, Çevik Ö, Ercan F, Yeğen BÇ. Nesfatin-1 ameliorates testicular injury and supports gonadal function in rats induced with testis torsion. Peptides. 2018,107:1-9.

33. Zhang J, Li S, Li L, Li M, Guo C, Yao J, Mi S. Exosome and exosomal microRNA: trafficking, sorting, and function. Genomics Proteomics Bioinformatics. 2015,13(1):17-24.

34. Halladin NL. Oxidative and inflammatory biomarkers of ischemia and reperfusion injuries. Dan Med J. 2015,62(4):B5054.

35. Fernández V, Tapia G, Varela P, Gaete L, Vera G, Mora C, Vial MT, Videla LA. Causal role of oxidative stress in liver preconditioning by thyroid hormone in rats. Free Radic Biol Med. 2008,44(9):1724-31.

36. Turner TT, Bang HJ, Lysiak JL. The molecular pathology of experimental testicular torsion suggests adjunct therapy to surgical repair. J Urol. 2004,172(6 Pt 2):2574-8.

37. Das A, Sinha M, Datta S, Abas M, Chaffee S, Sen CK, Roy S. Monocyte and macrophage plasticity in tissue repair and regeneration. Am J Pathol. 2015,185(10):2596-606.

38. Shapouri-Moghaddam A, Mohammadian S, Vazini H, Taghadosi M, Esmaeili SA, Mardani F, Seifı B, Mohammadi A, Afshari JT, Sahebkar A. Macrophage plasticity, polarization, and function in health and disease. J Cell Physiol. 2018,233(9):6425-40.

39. Oishi Y, Manabe I. Macrophages in inflammation, repair and regeneration. Int Immunol. 2018,30(11):511-28.

40. Heo JS, Choi Y, Kim HO. Adipose-Derived Mesenchymal Stem Cells Promote M2 Macrophage Phenotype through Exosomes. Stem Cells Int. 2019,2019:7921760.

41. Deng S, Zhou X, Ge Z, Song Y, Wang H, Liu X, Zhang D. Exosomes from adipose-derived mesenchymal stem cells ameliorate cardiac damage after myocardial infarction by activating S1P/SK1/S1PR1 signaling and promoting macrophage M2 polarization. Int J Biochem Cell Biol. 2019,114:105564.

42. Zhao H, Shang Q, Pan Z, Bai Y, Li Z, Zhang H, Zhang Q, Guo C, Zhang L, Wang Q. Exosomes From Adipose-Derived Stem Cells Attenuate Adipose Inflammation and Obesity Through Polarizing M2 Macrophages and Beiging in White Adipose Tissue. Diabetes. 2018,67(2):235-47.

\section{Tables}

Table 1. Johnsen's score for spermatogenic founction 


\section{Score Characteristics}

10 Complete spermatogenesis with many spermatozoa

9 Many spermatozoa present but germinal epithelium disorganized with marked sloughing or obliteration

$8 \quad$ Only few spermatozoa ( $(5-10)$ present in section

$7 \quad$ No spermatozoa but many spermatids present

$6 \quad$ No spermatozoa and only few spermatids ( $(5-10)$ present

$5 \quad$ No spermatozoa, no spermatids but several or many spermatocytes present

$4 \quad$ Only few spermatocytes ( $(5)$ and no spermatids or spermatozoa present

3 Spermatogonia are the only germ cells present

2 No germ cells but Sertoli cells are present

$1 \quad$ No cells in tubular section

\section{Figures}

A

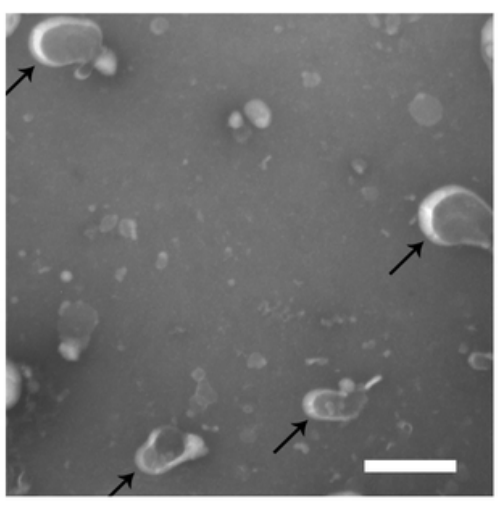

C

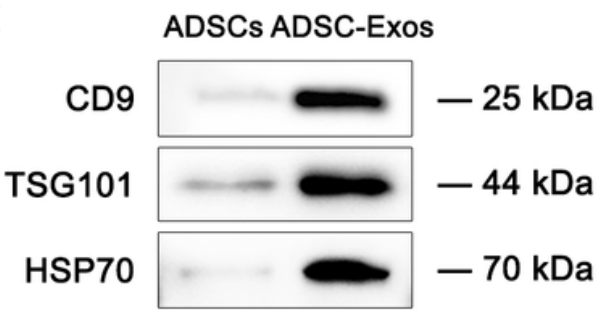

B

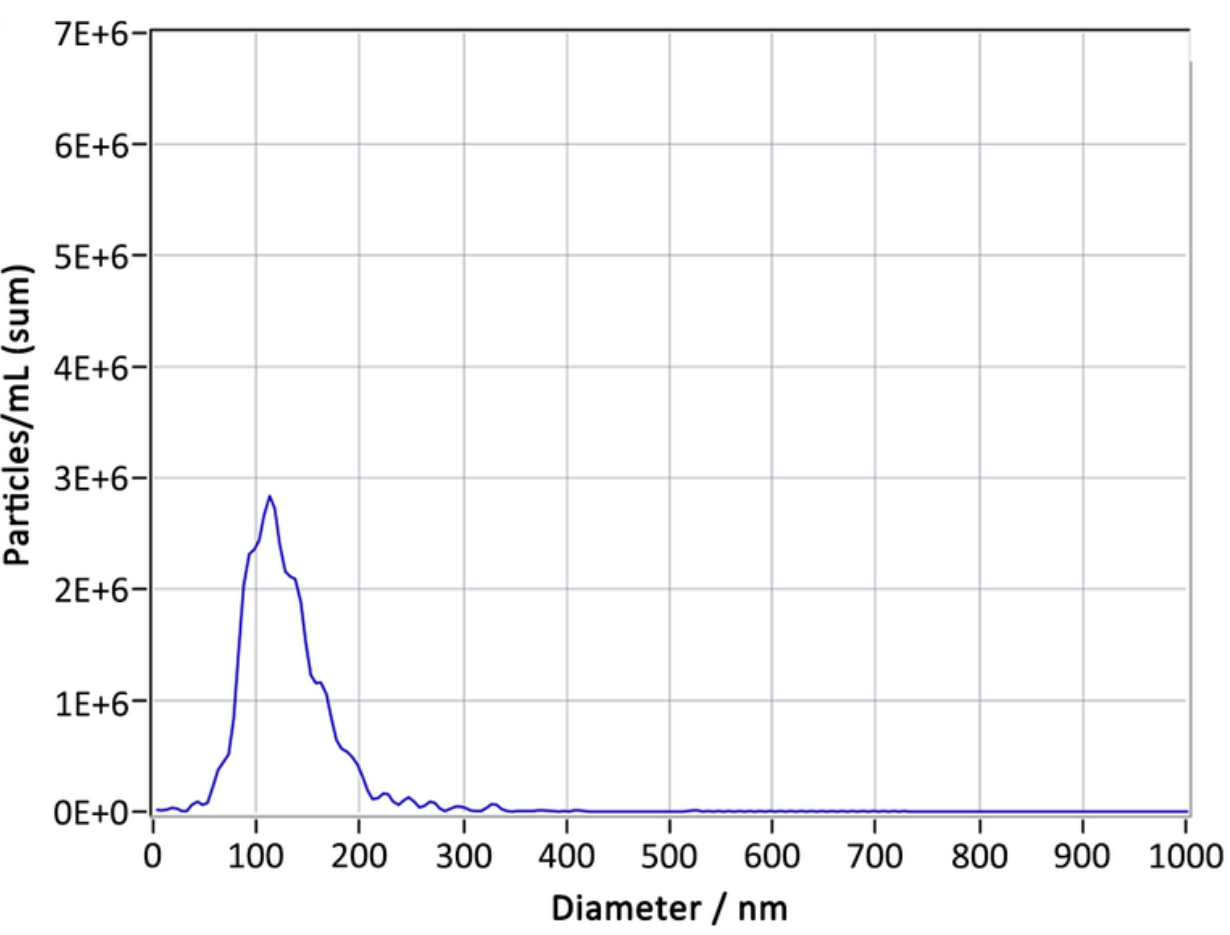

Figure 1 
Characterization of ADSC-Exos. A Morphology of ADSC-Exos under a transmission electron microscope. B Particle size distribution. C Western blot was used to detect exosome surface markers. Bars, $100 \mathrm{~nm}$

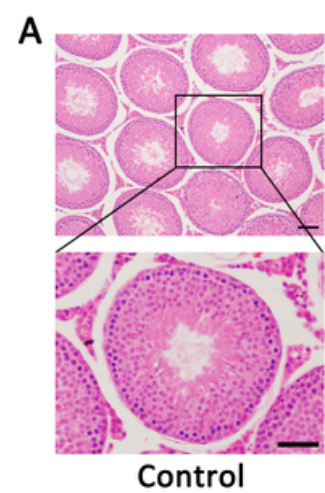

C

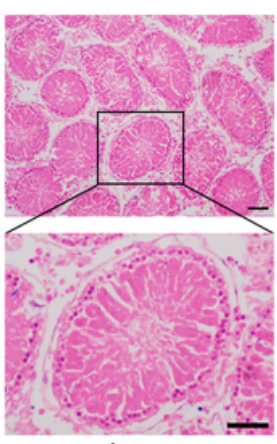

I/R-D3

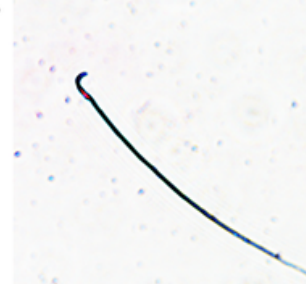

Normal

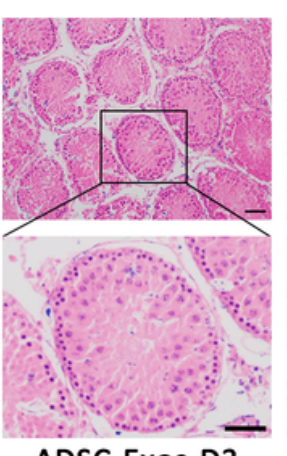

ADSC-Exos-D3

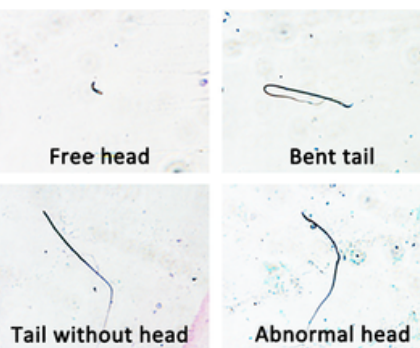

$\mathbf{F}$

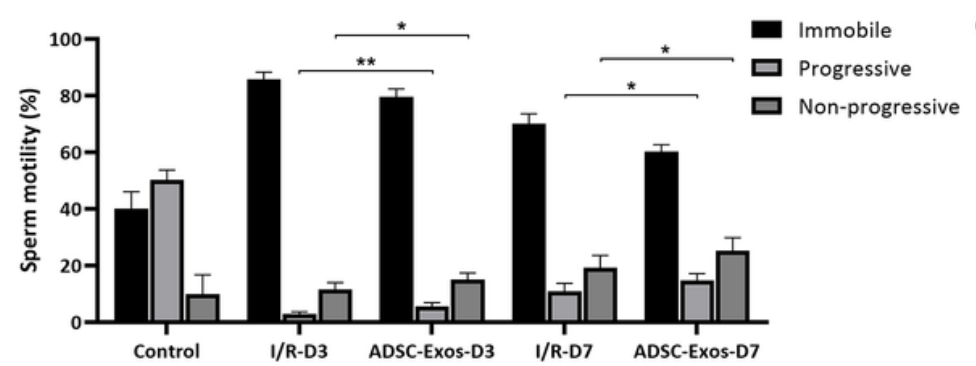

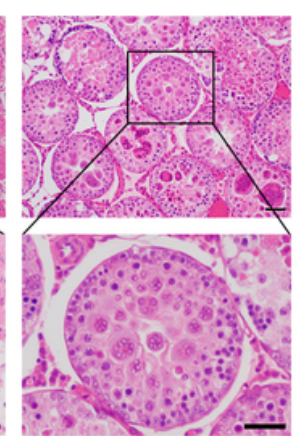

I/R-D7
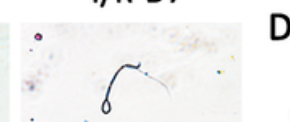

Coiled tail

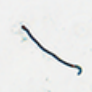

Short tail

G

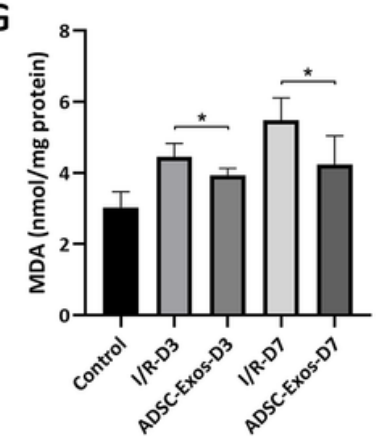

B

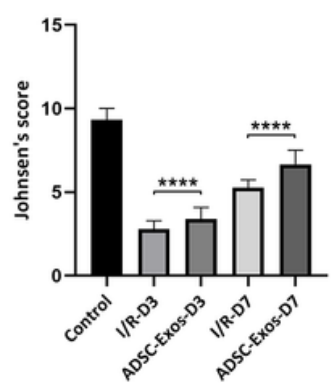

E
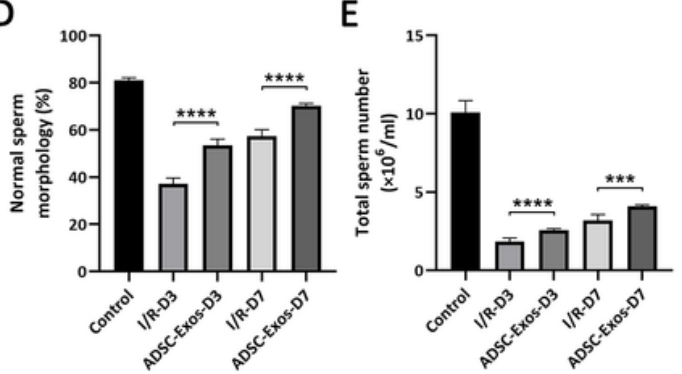

$\mathrm{H}$

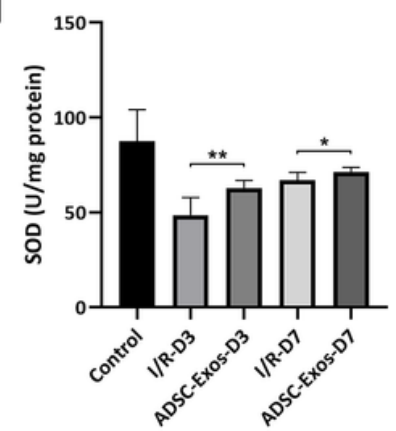

Figure 2

ADSC-Exos alleviate testicular torsion-detorsion injury. A,B The H\&E staining after torsion-detorsion injury at day $3(n=6)$ and day $7(n=6)$. C Sperm with normal and abnormal morphology. D-F Results of sperm parameters (quantity, morphology and motility) at day $3(n=6)$ and day $7(n=6) . G, H$ Results of Biochemical analysis (MDA and SOD) at day $3(n=6)$ and day $7(n=6)$. Bars, $100 \mu$ m. Data are represented as mean \pm SD. ${ }^{*} P<0.05,{ }^{*} P<0.01, * \star * P<0.001, * \star \star \star P<0.001$ 


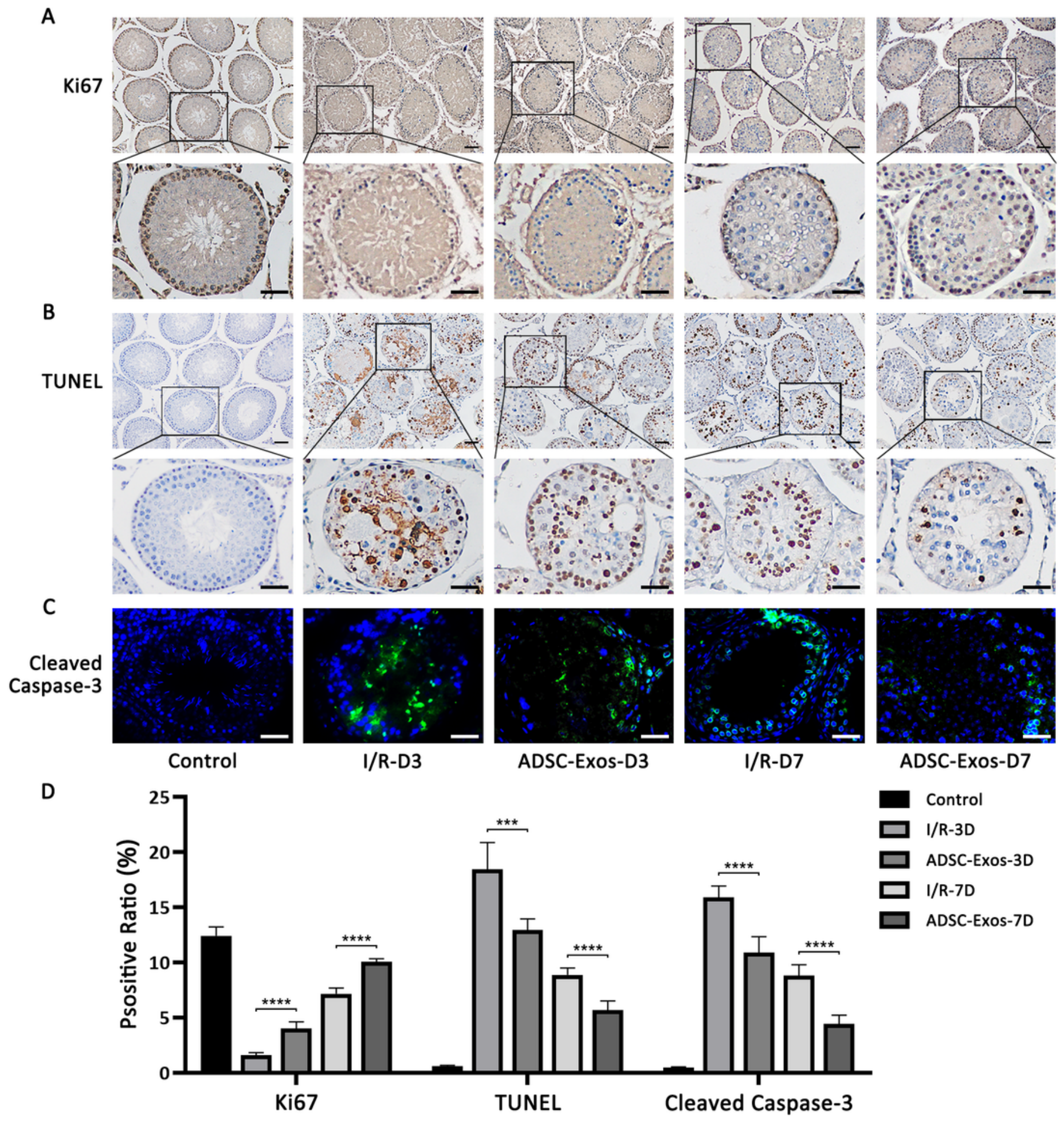

Figure 3

ADSC-Exos promote spermatogenic cells proliferation and inhibits apoptosis in vivo. A The expression of Ki67 was detected by immunohistochemistry at day $3(n=6)$ and day $7(n=6)$. B TUNEL assay of testicular tissues after torsion-detorsion injury at day $3(n=6)$ and day $7(n=6)$. C The expression of cleaved Caspase-3 was detected by Immunofluorescence at day $3(n=6)$ and day $7(n=6)$. D 
Quantitative analysis of Ki67, TUNEL and cleaved Caspase-3 expression. Bars (Ki67 and TUNEL), $100 \mu \mathrm{m}$, Bars (Cleaved Caspase-3), $50 \mu \mathrm{m}$. Data are represented as mean $\pm \mathrm{SD}$. ${ }^{\star \star *} \mathrm{P}<0.001$, ${ }^{\star \star *} \mathrm{P}<0.001$

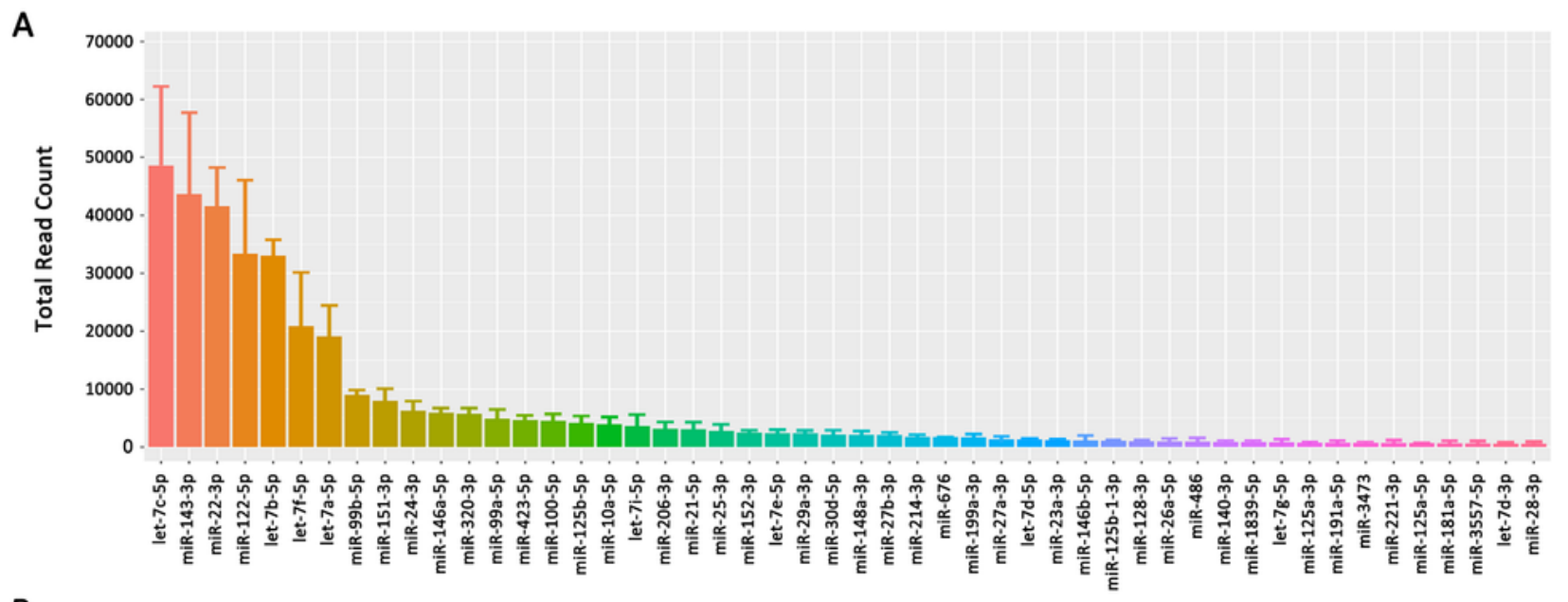

B

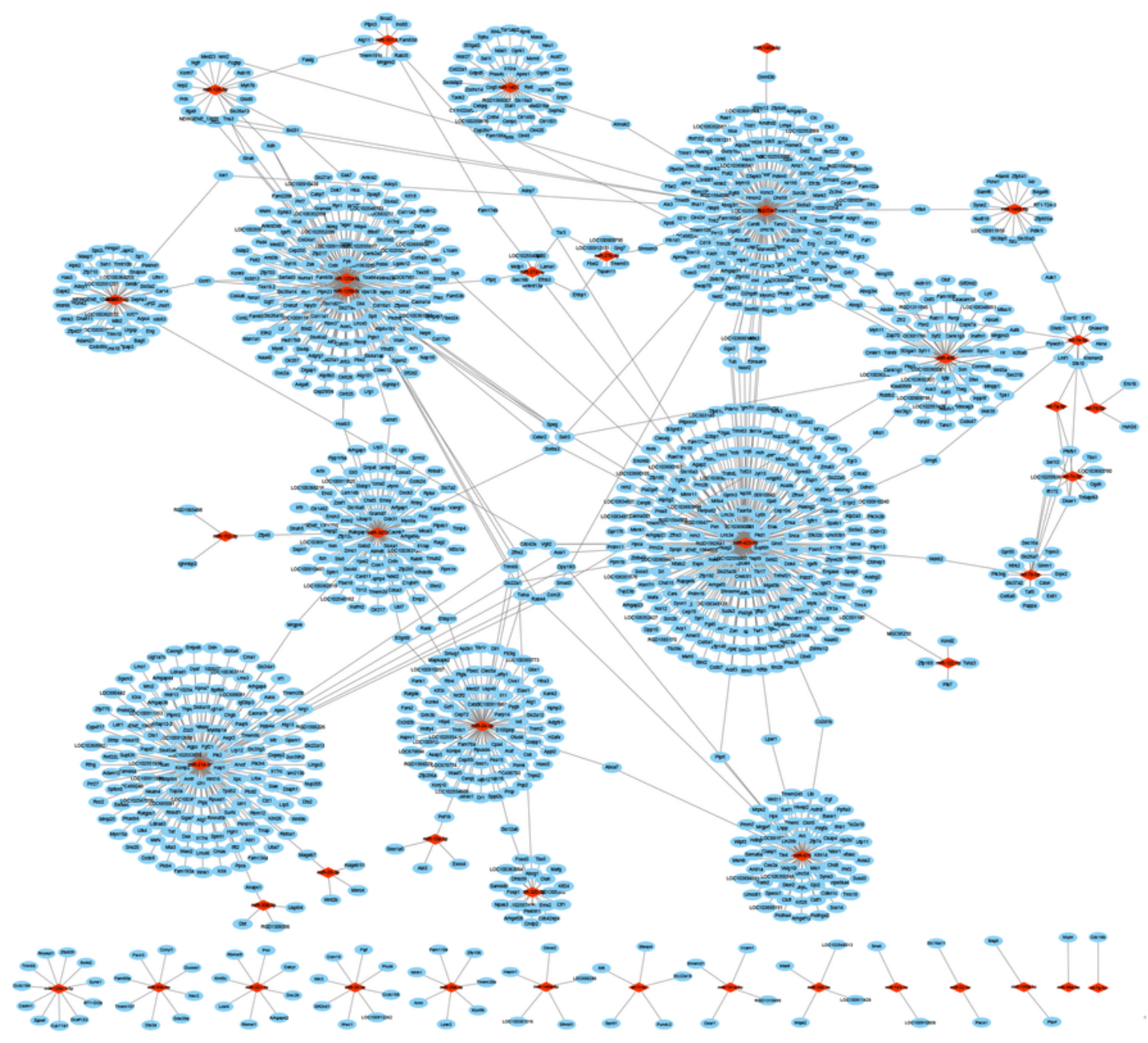

\section{Figure 4}

miRNA sequencing and target genes analysis of ADSC-Exos. A The top 50 miRNAs that were detected in ADSC-Exos $(n=3)$. B Prediction of miRNA target genes. 
A
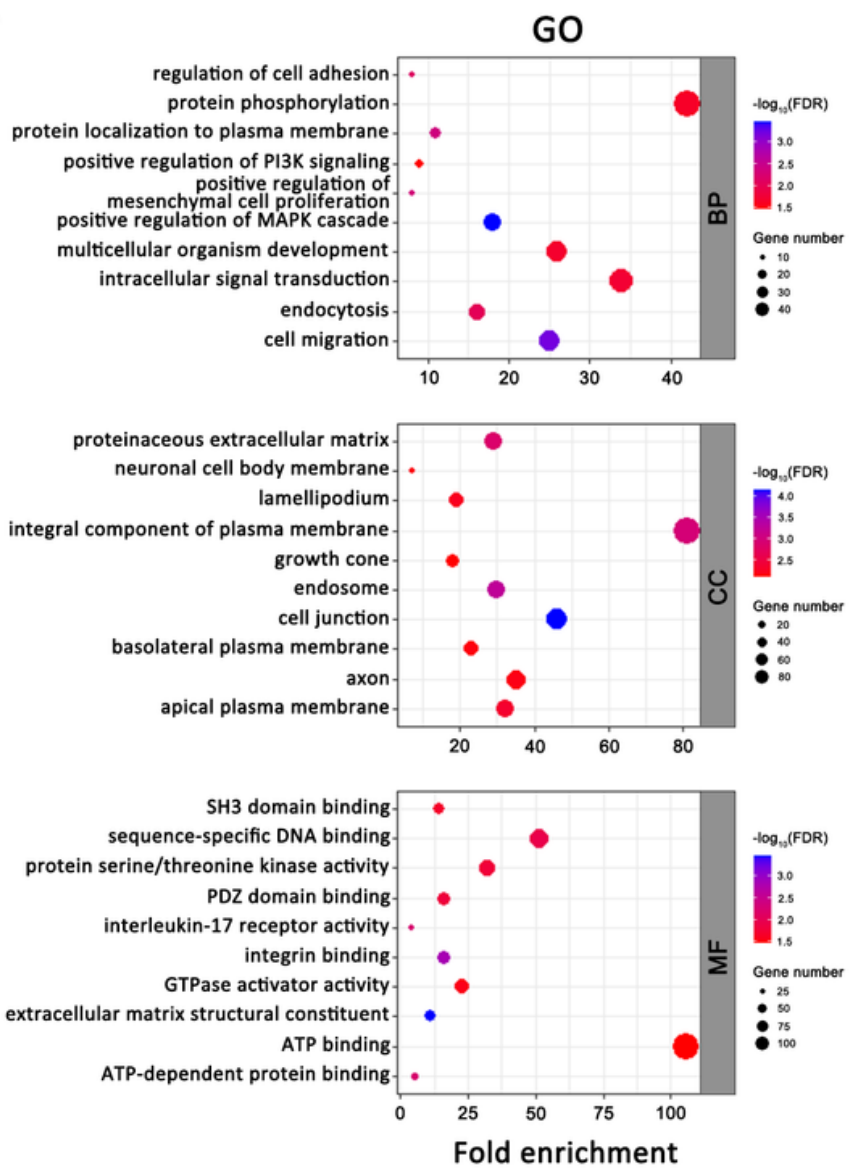

B

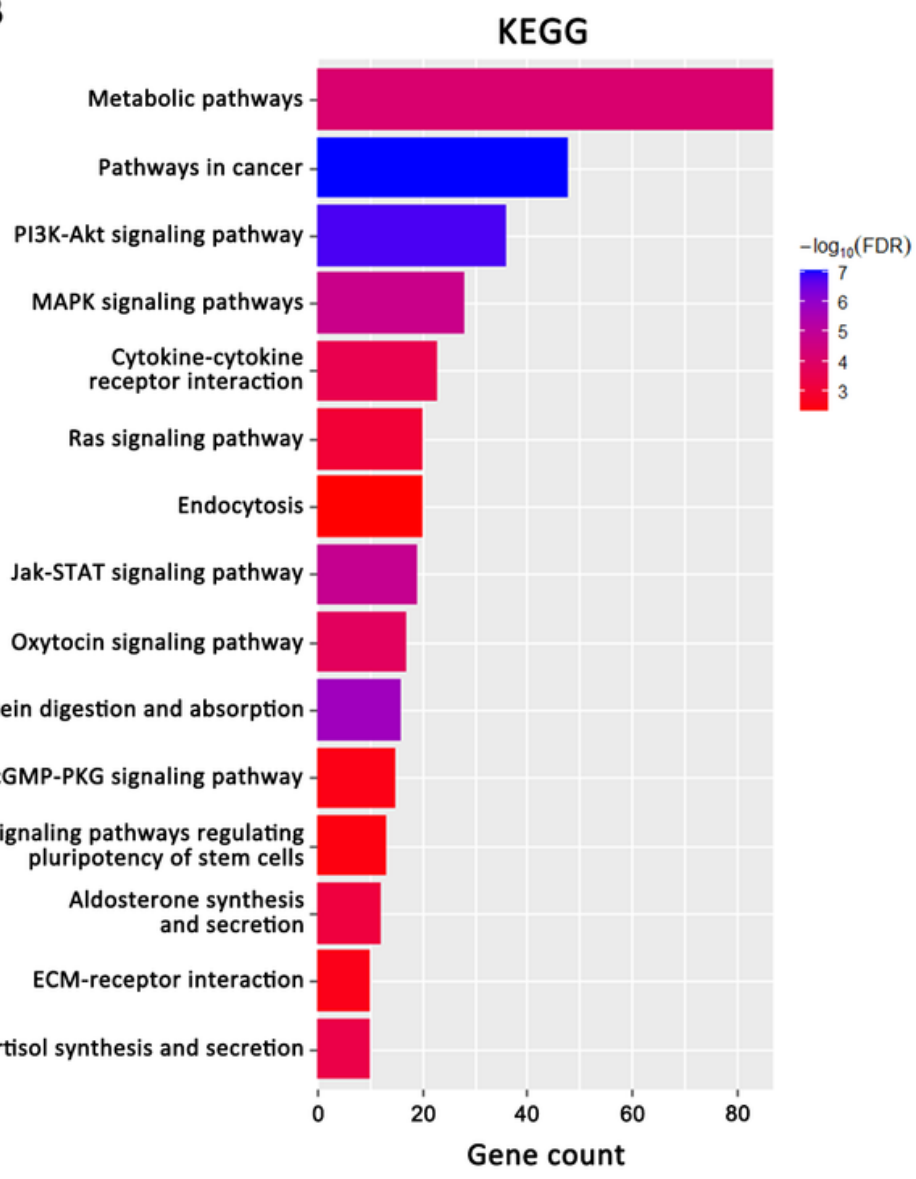

\section{Figure 5}

Bioinformatics analysis of ADSC-Exos target genes. A,B GO and KEGG pathway enrichment analyses of the possible target genes. 


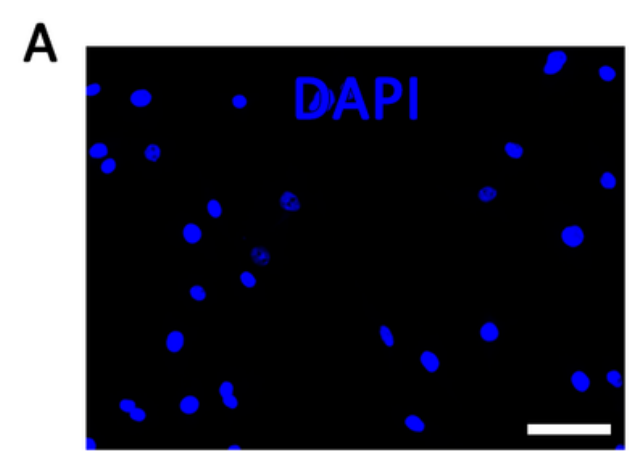

B

D
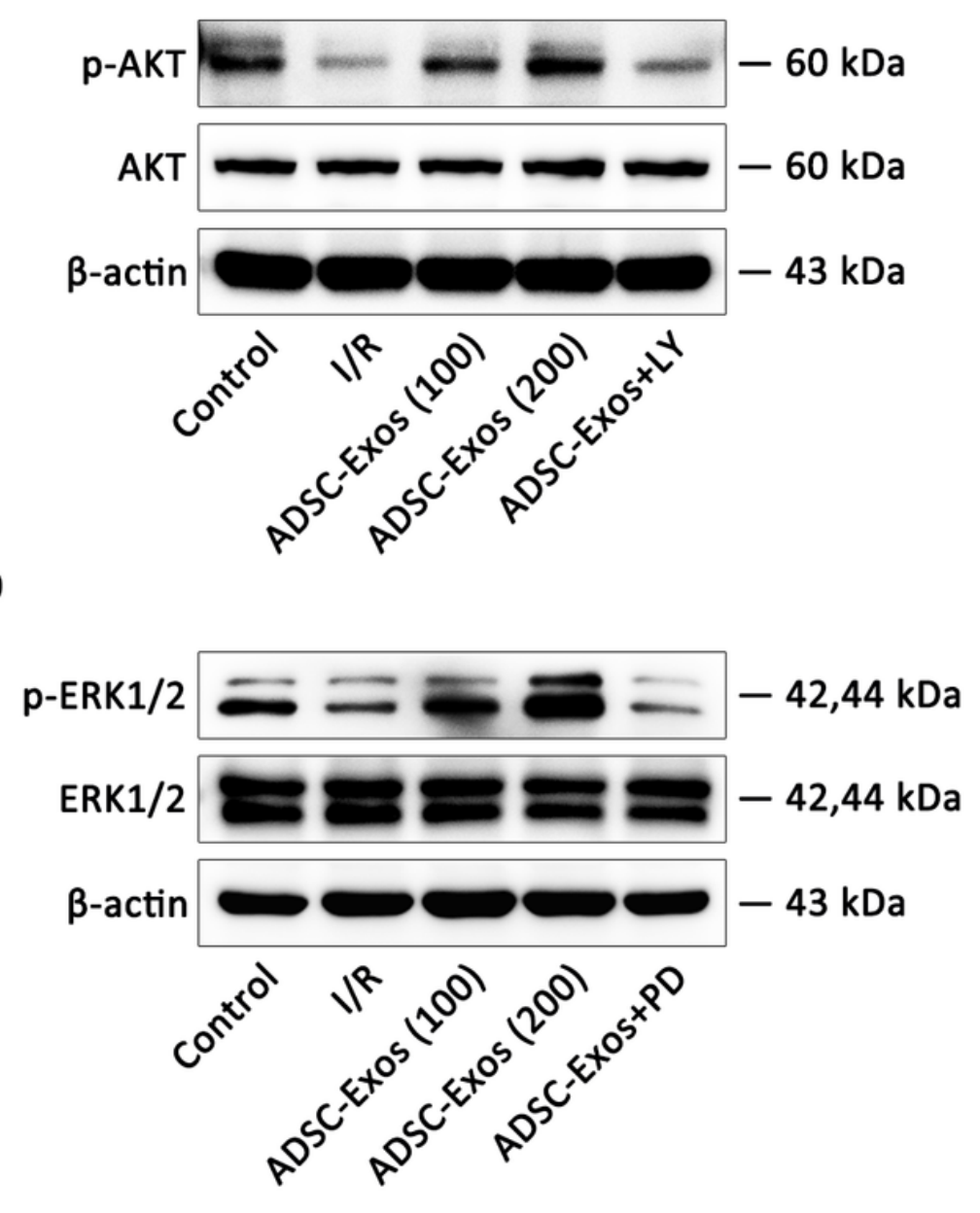

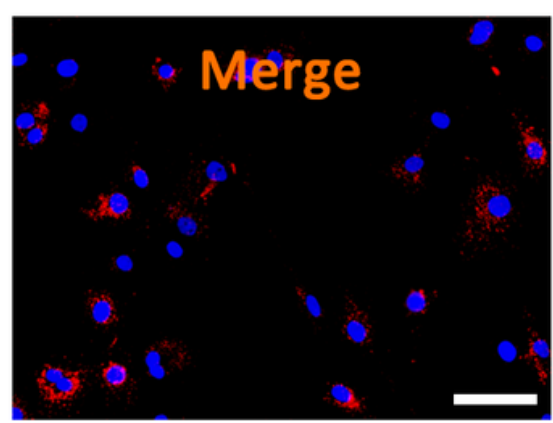

C

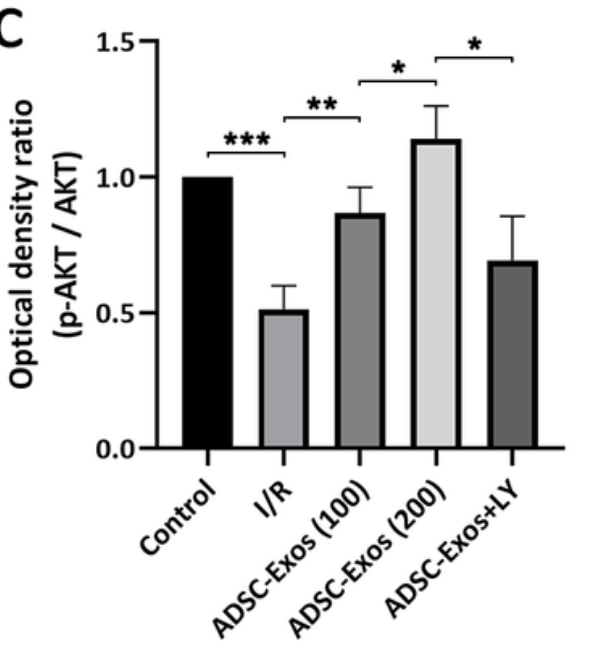

E

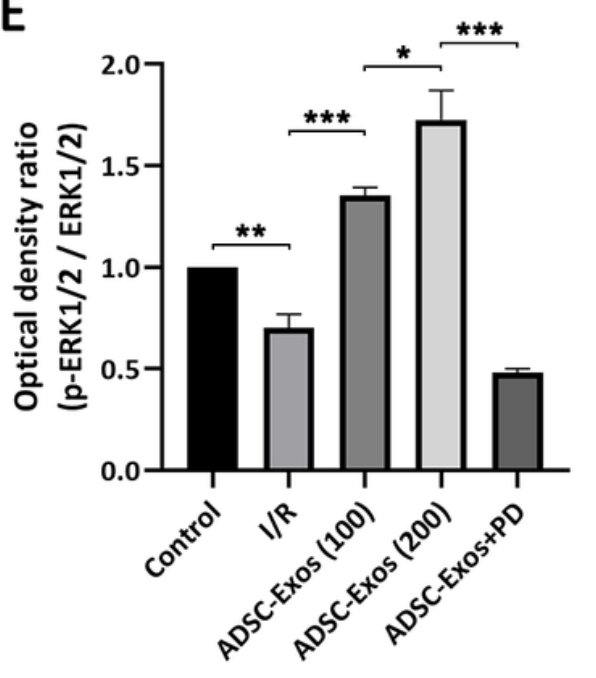

Figure 6

ADSC-Exos activate PI3K / AKT and MAPK / ERK1/2 signaling pathways in GC-1 spg cells. A PKH26labeled ADSC-Exos internalization by GC-1 spg cells. B-E Western blot analysis of protein levels of p-AKT and $\mathrm{p}$-ERK induced by different concentrations of ADSC-Exos or pathway inhibitors. Bars, $100 \mu \mathrm{m}$. Data are represented as mean $\pm S D$. ${ }^{*} P<0.05,{ }^{*} \mathrm{P}<0.01, * \star \star P<0.001$ 

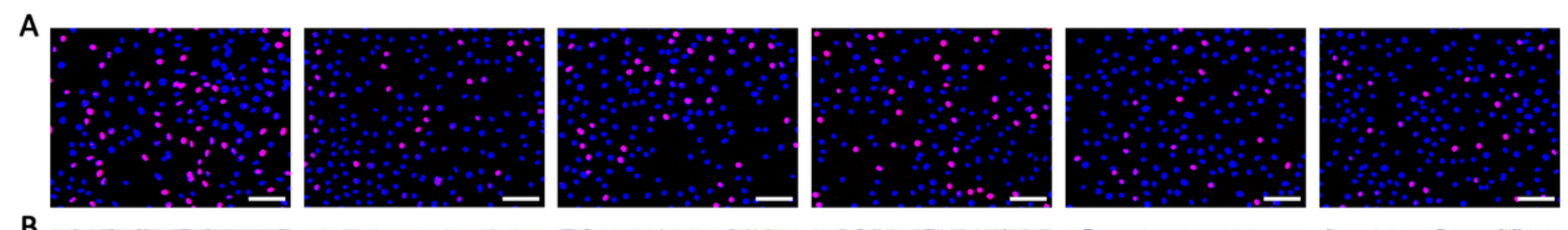

B

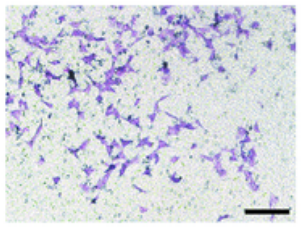

C
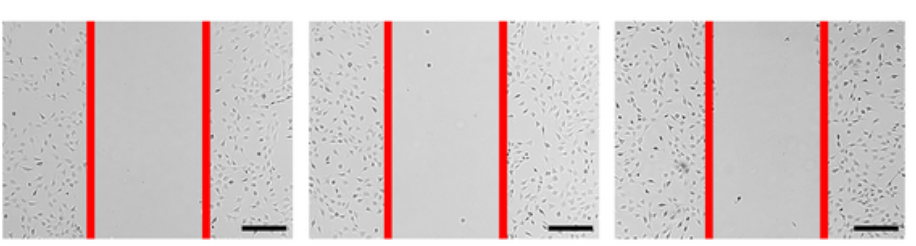

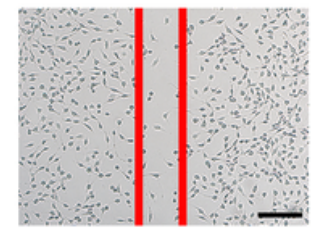

Control

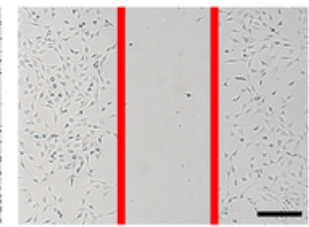

I/R

D

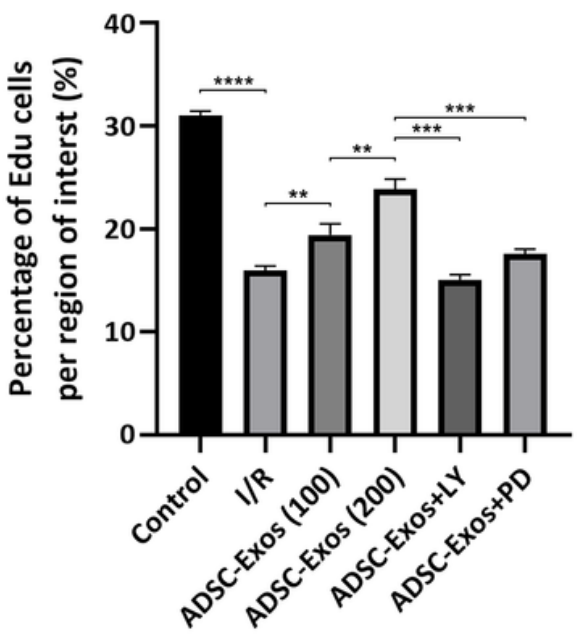

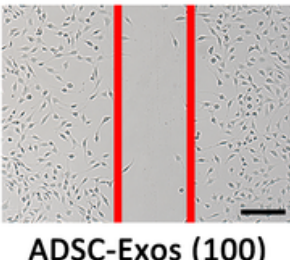

E

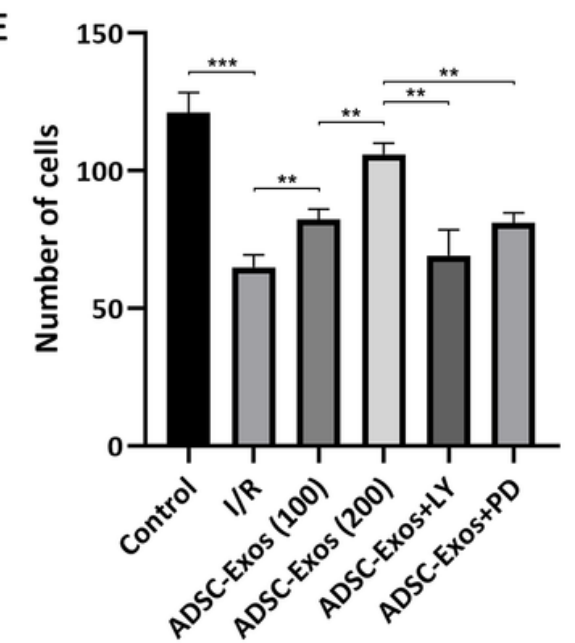

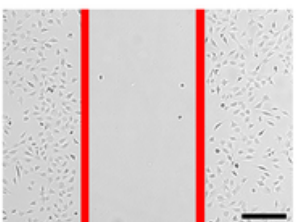
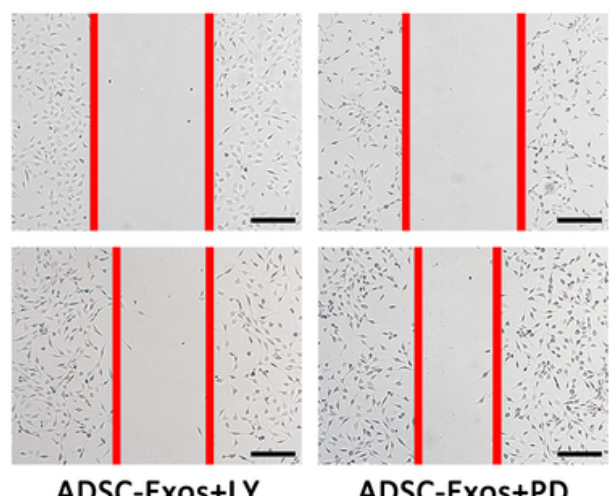

ADSC-Exos+PD

$F$

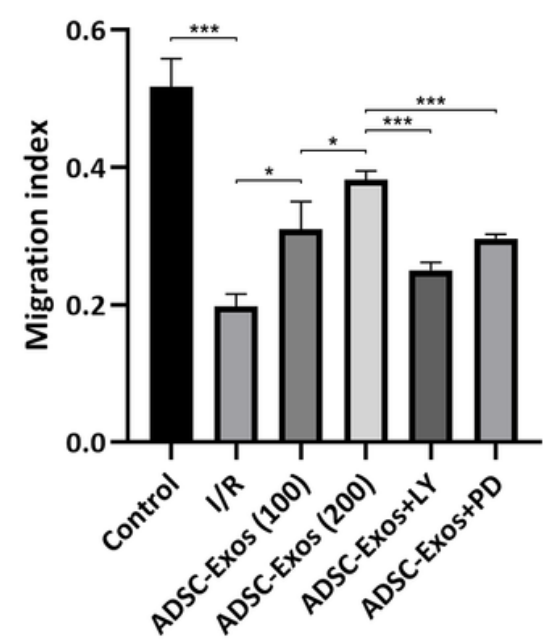

Figure 7

ADSC-Exos promote the proliferation and migration of spermatogenic cells after I/R injury A,D Effect of ADSC-Exos on the proliferation of GC-1 spg cells by EdU assays. B,C,E,F Effect of ADSC-Exos on the migration of GC-1 spg cells by transwell assays and scratch test. Bars, $100 \mu \mathrm{m}$. Data are represented as

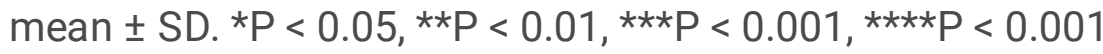




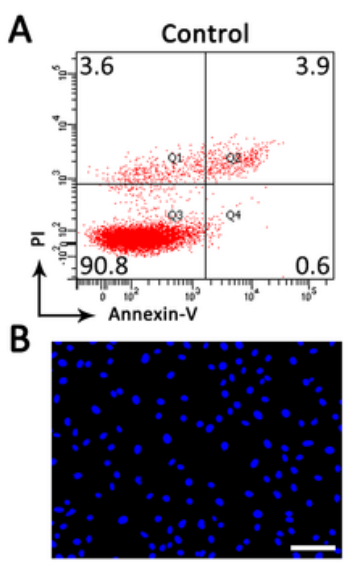

Control

C

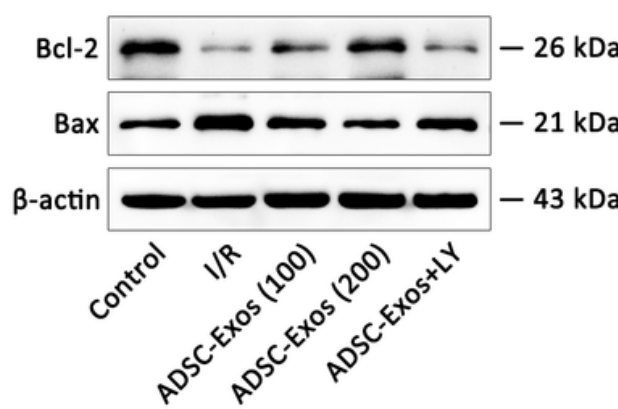

G

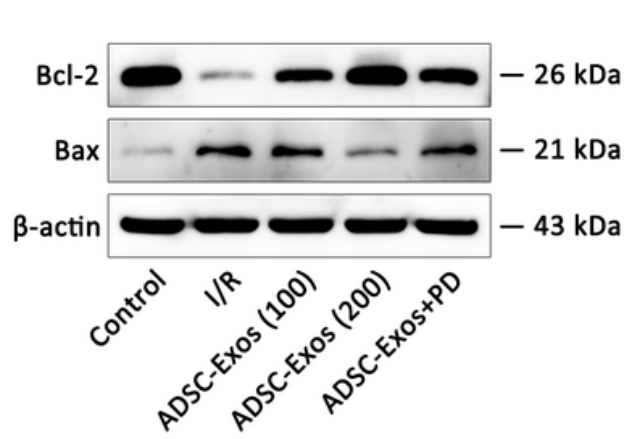

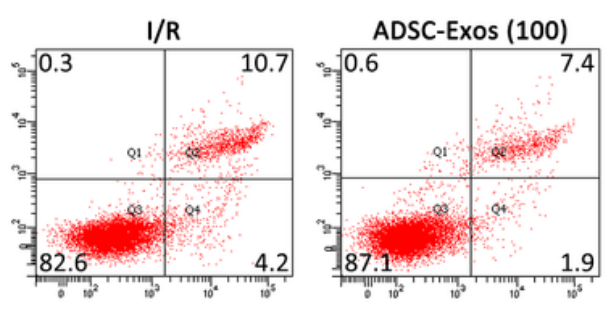

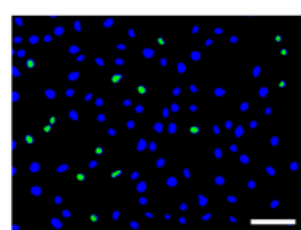

I/R

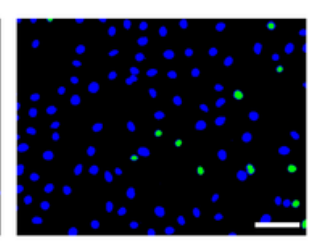

ADSC-Exos (100)

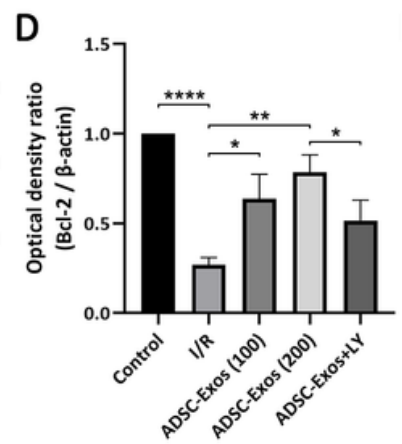

$\mathrm{H}$

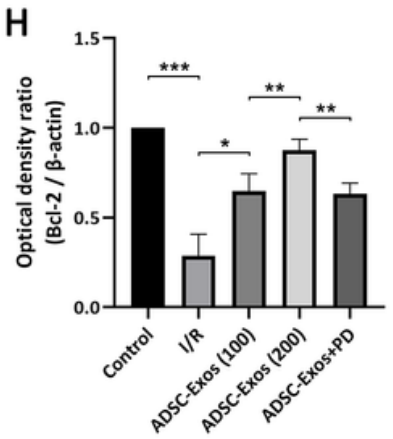

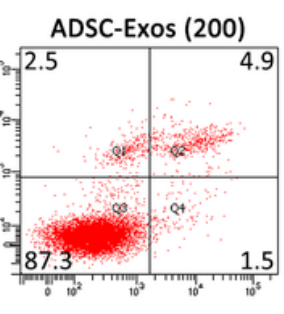
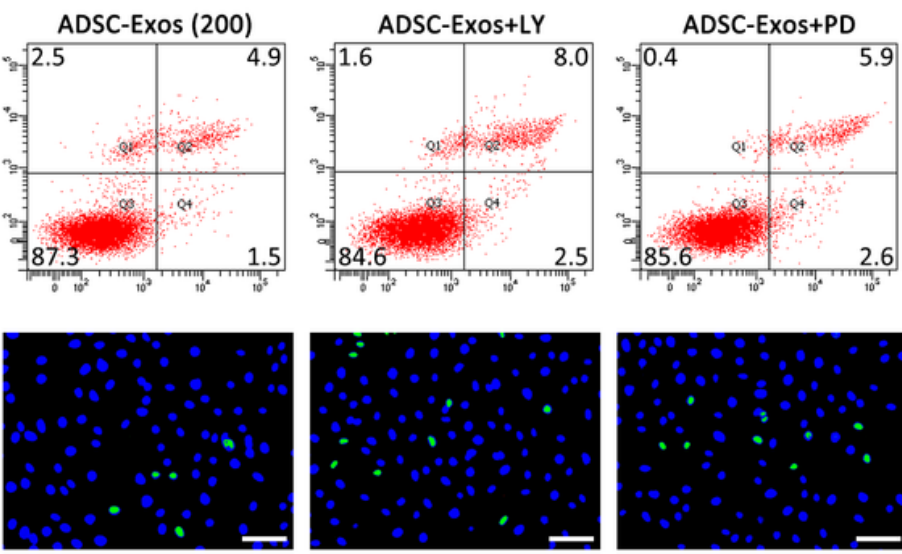

ADSC-Exos+LY

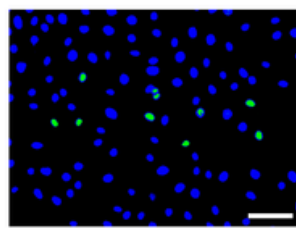

ADSC-Exos+PD
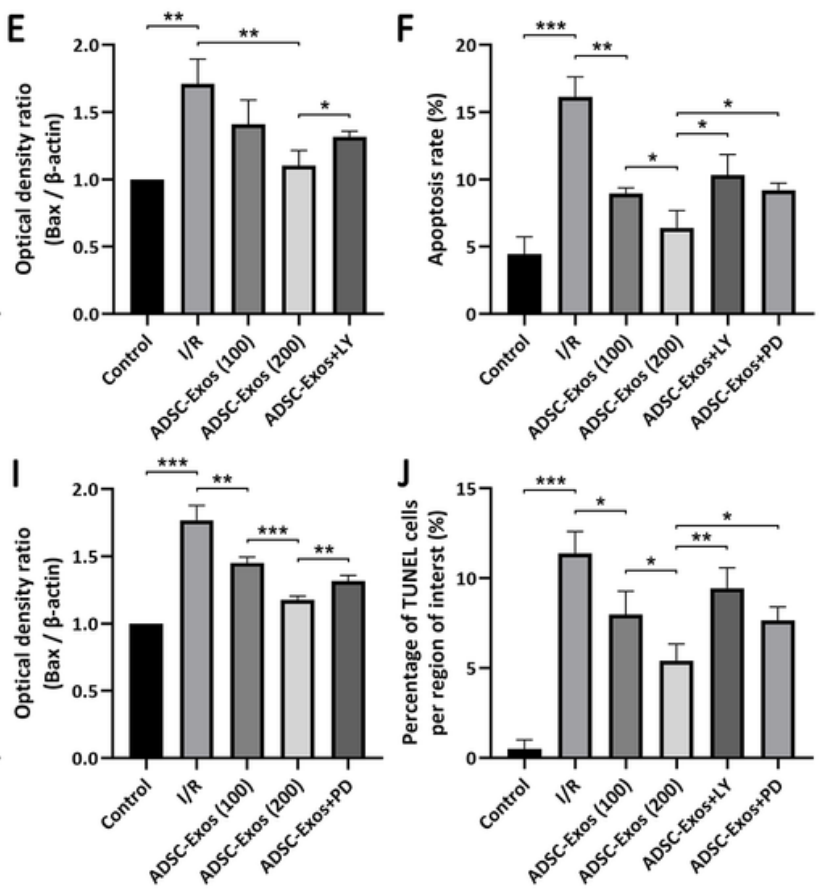

Figure 8

ADSC-Exos inhibit apoptosis of spermatogenic cells after l/R injury A,B,F,J Effect of ADSC-Exos on apoptosis of GC-1 spg cells by flow cytometry analysis and TUNEL staining. C-E,G-I Western blot analysis of protein levels of Bcl-2 and Bax induced by ADSC-Exos or pathway inhibitors. Bars, $100 \mu \mathrm{m}$. Data are represented as mean $\pm S D$. ${ }^{\star} P<0.05,{ }^{*} \mathrm{P}<0.01$, $* \star * P<0.001, * \star \star \star P<0.001$ 


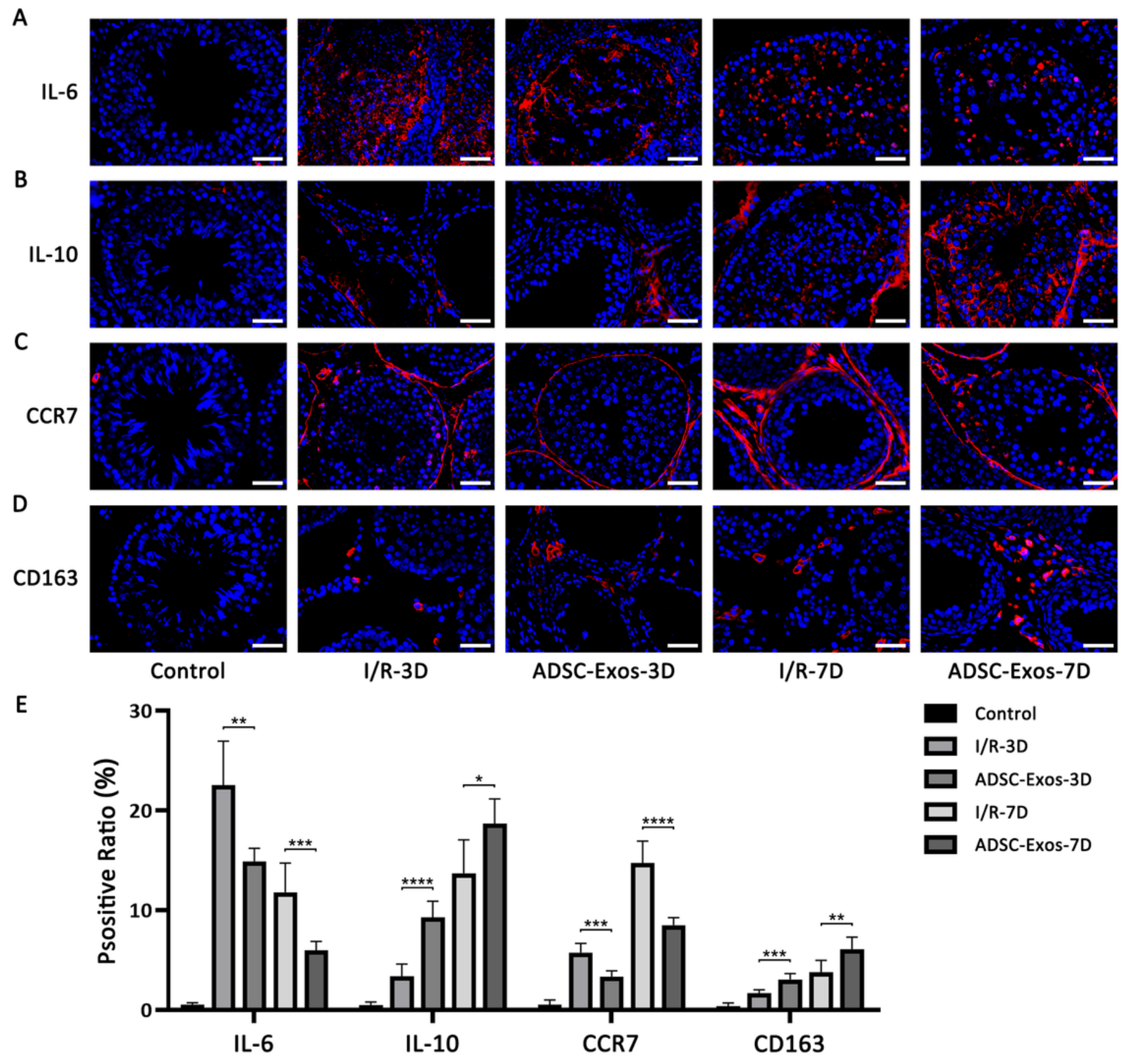

Figure 9

ADSC-Exos inhibits inflammatory expression induced by testicular torsion-detorsion injury. A-D The expression of IL-6, IL-10, CCR7 and CD163 were detected by Immunofluorescence at day $3(n=6)$ and day $7(n=6)$. E Positive ratio of inflammation-related factors $(n=6)$. Bars, $50 \mu \mathrm{m}$. Data are represented as

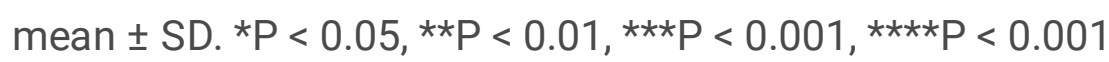

\section{Supplementary Files}

This is a list of supplementary files associated with this preprint. Click to download. 
- GA.tif

Page 25/25 